\section{PTU-092 THE MANAGEMENT OF ALCOHOL WITHDRAWAL IN PATIENTS WITH ADVANCED LIVER DISEASE}

doi:10.1136/gutjnl-2013-304907.182

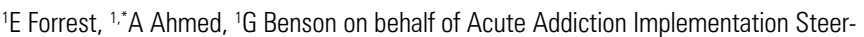
ing Group, Glasgow. 'Glasgow Royal Infirmary, Glasgow, UK

Introduction Alcohol withdrawal syndrome (AWS) is a common reason for hospital admission. However a significant number of these patients have co-existent liver disease or other medical problems. There is little information regarding the management of these patients. Methods Patients were assessed for hazardous drinking using the FAST score. Data was collected prospectively on FAST positive patients with regards to their subsequent treatment using a unified AWS guideline. Patients with known liver disease or presenting with decompensated liver disease were identified (Group 1) and compared with patients admitted with non-hepatic primary alcohol-related diagnoses (Group 2) and patients with admitted primarily non-alcohol related medical diagnoses (Group 3). Results are expressed as median (95\% confidence).

Results 53 patients had significant liver disease (Group 1), with 153 in Group 2 and 106 in Group 3. Median MELD score in Group 1 was $15.4[12.8,17.6]$. The three groups had similar FAST scores: 14 $[12,15], 14[13,14]$ and $13[12,14]$ respectively. Fewer patients in Group 1 and Group 3 required benzodiazepine (BZD) treatment compared with Group 2. When required, the median BZD prescription (mg diazepam equivalent) during admission was greater for Group 2 patients compared with Group 1 and Group 3 (Table). More patients in Group 1 were treated with lorazepam rather than diazepam; $13 \%$ of $5 \%$ in Group $2(p=0.1,-1,20.4)$ and $3 \%$ in Group 3 ( $p=0.04 ; 0.7,22.3)$. The proportion of patients requiring parenteral treatment for breakthrough symptoms were similar: Group 1: 5.6\%, Group 2: 4.6\%, Group 3 4.7\%.

\section{Abstract PTU-092 Table}

\begin{tabular}{lll}
\hline & Not Requiring BZD & Median BZD use (mg diazepam equiv) \\
\hline Group 1 & $14(26 \%)^{*}$ & $70(48,111) \$$ \\
Group 2 & $16(10 \%)^{*} \#$ & $130(105,160 \$ \sim)$ \\
Group 3 & $35(33 \%) \#$ & $40(30,80) \sim$ \\
\hline${ }^{*} p=0.008(3.5,30.5) ; \# p<0.0001(12.4,33.7) \cdot \$ p=0.003 ; \sim p=0.0001$
\end{tabular}

There were correlations between the FAST score and subsequent amount of diazepam prescribed for Group $2(p=0.002 ; 0.09,0.4)$ and Group 3 ( $p=0.03 ; 0.02,0.41)$, but not for Group 1 ( $p=0.26$; $-0.12,0.43$ ). Overall survival until 33 months was less in Group 1 (64\%) compared with Group 2 (84\%; p = 0.0007 HR 0.29 [0.14, 0.60] and Group 3 (81\%; $p=0.016$ HR $2.30[1.17,4.55])$.

Conclusion Compared to patients admitted with primarily AWS or alcohol related seizures, patients with chronic liver disease and those with other medical problems were less likely to require any benzodiazepine therapy and require lesser amounts of such treatment. The expected association between indicators of harmful/ dependent drinking and BZD requirement was lost in liver disease patients. Care should be taken to avoid unnecessary over-treatment of these patients.

Disclosure of Interest None Declared

\section{PTU-093 HCC DIAGNOSED ON SURVEILLANCE PROGRAMIMES: IMPACT ON STAGE AND OUTCOME}

doi:10.1136/gutjnl-2013-304907.183

1."A Ahmed, ${ }^{2} \mathrm{Z}$ Mustafa, ${ }^{2} \mathrm{M}$ Neilson, ${ }^{2} \mathrm{M}$ Rutherford, ${ }^{3} \mathrm{~S}$ Ballantyne, ${ }^{3} \mathrm{R}$ Kasthuri, ${ }^{4} \mathrm{~J}$ Evans, ${ }^{2} \mathrm{E} H$ Forrest, ${ }^{2} \mathrm{~S}$ Barclay, ${ }^{2} \mathrm{R}$ Gillespie, ${ }^{5} \mathrm{M}$ Priest, ${ }^{5} \mathrm{P}$ R Mills, ${ }^{2} \mathrm{~A} J$ Stanley 'Gastroenterology, Victoria Infirmary; ${ }^{2}$ Gastroenterology, Glasgow Royal Infirmary; ${ }^{3}$ Radiology, Gartnavel General Hospital; ${ }^{4}$ Beatson Oncology Centre; ${ }^{5}$ Gastroenterology, Gartnavel General Hospital, Glasgow, UK
Introduction Surveillance of cirrhotic patients for HCC is recommended by numerous national and international guidelines. However many patients are still diagnosed de novo with this malignancy. Data on the benefits of surveillance remains relatively limited. Our aim was to compare stage at diagnosis and patient outcome for those diagnosed on surveillance and those who were not.

Methods Using our regional HCC MDT database, we analysed patients diagnosed with HCC between January 2009 and January 2012. All patients were staged using the Barcelona Clinic Liver Cancer (BCLC) system. We compared the stage at diagnosis, the treatment strategy after MDT discussion, and the survival in those diagnosed in surveillance with those diagnosed de novo. Statistical comparisons were made using CHI-squared or Kaplan Meier analysis as appropriate.

Results 190 patients were diagnosed with HCC at MDT during the study period. We had full follow-up data on 169 patients which were used for analyses, with mean follow up 10 months. Mean age was 68 years and $82 \%$ patients were male. Aetiology was alcoholic liver disease in $32 \%$ and $\mathrm{HCV}$ in $15 \% .38(22 \%)$ patients were in surveillance programmes at diagnosis of HCC and 132 (78\%) were not. Tumours were BCLC stage A at diagnosis in $28.9 \%$ patients in surveillance, compared with $6.1 \%$ not in surveillance $(p=0.0003)$. $15.8 \%$ those diagnosed in surveillance underwent transplantation or resection, compared with $2.3 \%$ who were not $(p=0.004)$. Survival for those diagnosed in surveillance was greater than those diagnosed de novo $(p=0.01)$

Conclusion Most patients diagnosed with HCC in our region were not in surveillance programmes. Patients diagnosed on surveillance were more likely to have potentially curative disease and had higher overall survival.

Disclosure of Interest None Declared

\section{REFERENCES}

EASL Clinical practise guidelines: Management of hepatocellular carcinoma. J Hepatol 2012; 56:908-943

AASLD Practice Guidelines management of Hepatocellular Carcinoma: An update Hepatol 2010; 53(3):1021-1022

APASL consensus on HCC. Hepatol Intern 2010; 4:439-74

\section{PTU-094 SHOULD LIVER BIOPSIES BE REPORTED BY PATHOLOGISTS WITH A SUBSPECIALIST INTEREST IN HEPATOLOGY?}

doi:10.1136/gutjnl-2013-304907.184

1,*B Krishnan, 'M Stares, 'H Rajabally, 'R D'Souza. 'Gastroenterology, Chasefarm Hospital, London, UK

Introduction Histopathologists working in a district general hospital usually do not have a subspecialist interest in hepatology. Most district general hospitals have a gastroenterology service and local pathologists usually report liver biopsies. The Royal College of Pathologist (RCP) recommend that 'as minimal acceptable practise' a liver biopsy report should include the clinical diagnosis, biopsy size, overall architecture, degree of fibrosis, severity in chronic liver disease (staging/grading), a definitive diagnosis or discussion of the differential diagnosis. Appropriate negative findings (e.g. lack of iron overload or alpha-1-antitrypsin globules) should be documented in the report. Methods A retrospective analysis of all liver biopsies between January 2010 to February 2012 at two district general hospitals (Barnet and Chasefarm NHS trust) in North London was performed. Data was collected from medical records and electronic results. Our aim was to assess whether liver biopsies provided the clinician with adequate information about diagnosis.

Results 107 liver biopsies were performed during this period under ultrasound guidance by a radiologist. Mean patient age was 62 years (Range 19 -90). The mean number of core biopsies per patient was 1.5 (range 1 - 6). 10.7\% (10/107) of the report did not mention a clinical diagnosis. 30\% (32/107) of the biopsy report did not have a 